\title{
Intestinal obstruction from multifocal endometriosis: A case report and review of literature
}

\author{
Babatunde M. Duduyemi ${ }^{1}$, Osei Owusu-Afriyie ${ }^{2}$, Prince Bohene ${ }^{3}$, Akinwale N. Titiloye ${ }^{1}$, \\ Sampene P. Ossei ${ }^{1}$, Ernest K. Adjei ${ }^{1}$, Babarinde A. Ojo ${ }^{4}$ \\ ${ }^{1}$ Department of Pathology, Kwame Nkrumah University of Science \& Technology, Kumasi, Ghana \\ ${ }^{2}$ Department of Pathology, Komfo Anokye Teaching Hospital, Kumasi, Ghana \\ ${ }^{3}$ University Teaching Hospital, Kwame Nkrumah University of Science \& Technology, Kumasi, Ghana \\ ${ }^{4}$ Department of Anatomical Pathology, Benue State University, Markudi, Nigeria
}

\section{Email address:}

babsdudu@yahoo.com (B. M. Duduyemi)

\section{To cite this article:}

Babatunde M. Duduyemi, Osei Owusu-Afriyie, Prince Bohene, Akinwale N. Titiloye, Sampene P. Ossei, Ernest K. Adjei, Babarinde A. Ojo. Intestinal Obstruction from Multifocal Endometriosis: A Case Report and Review of Literature. Journal of Surgery.

Vol. 2, No. 6, 2014, pp. 101-104. doi: 10.11648/j.js.20140206.15

\begin{abstract}
Endometriosis causing intestinal obstruction presents a difficult challenge in making a timely and accurate clinical diagnosis because of the non-specific nature of its presentation which can mimic other causes of intestinal obstruction. We report a case of large bowel obstruction and rectal bleeding secondary to multifocal intestinal endometriosis which was thought to be colorectal cancer. Patient had partial colectomy done and histology showed endometriosis. Intestinal endometriosis should be considered as a differential diagnosis in a young patient with recurrent lower abdominal pains and distension in conjunction with signs of obstruction.
\end{abstract}

Keywords: Endometriosis, Intestinal Obstruction, Nulliparous, Infertility

\section{Introduction}

Endometriosis is the term used to describe the presence of endometrial glands and/or stroma in an abnormal location outside the uterine cavity ${ }^{1}$. These endometrial cells are influenced by hormonal changes. Symptoms of endometriosis often present cyclically or worsen with the menstrual cycle $^{2}$. Endometriosis involving the intestine usually takes the form of asymptomatic, small, superficial serosal implants on segments of bowel lying in the pelvis in proximity to the genital organs ${ }^{3}$. Although statistics vary, it has been estimated that approximately $8 \%-15 \%$ of actively menstruating women have some degree of endometriosis ${ }^{4,5}$. Endometriosis affects the intestine in 3\%-34\% of cases and is generally asymptomatic or presents with non-specific gastrointestinal symptoms ${ }^{6,7,8,9}$. Intestinal obstruction due to endometriosis is rare. Intestinal obstruction most commonly occur secondary to adhesion, cancer, diverticulitis, volvulus or inflammatory bowel disease. Extensive intestinal wall involvement may result in obstruction and occasionally bleeding and requires distinction from a neoplasm or other inflammatory bowel process 5 . We present this rare case of acute large bowel obstruction with non-specific intestinal symptoms and difficulty in making a timely and accurate diagnosis.

\section{Case Report}

A 34 year old nulliparous patient seen at the outpatient department of the University Hospital of the Kwame Nkrumah University of Science \& Technology, Kumasi, Ghana having presented with recurrent abdominal pains for a month and lower abdominal distension of 5 days; with associated borborygmi, inability to pass faeces and flatus of five days duration, an episode of vomiting but no fever. No history of hematuria, or oliguria but she has been bleeding per vaginum 2 days before presentation. Examination revealed a young anxious looking lady, afebrile, well hydrated, and not pale or tachypnoiec with a pulse of $90 \mathrm{bpm}$, BP-124/80mmhg. She also came in with an upper gastrointestinal endoscopy result of pangastritis positive for H.pylori which was done 2 weeks prior to presentation. Examination of the abdomen revealed tender lower abdominal distension of about 15 -week gravid uterus, moves 
with respiration, no palpable organomegaly. Percussion over the lower abdomen was dull and bowel sound was hyperactive. A provisional diagnosis of intestinal obstruction with pangastritis was made and she was referred to the surgical outpatient unit. At the surgical outpatient a digital rectal examination revealed an empty anorectal pouch with no faecal matter palpable but a hard mass covered by mucosa but no bummer shelf. Vaginal examination showed a blood stained finger with a cervical os that barely admits a finger. There were no palpable masses on the vaginal wall.

Other systems were essentially normal. Urgent pregnancy test was negative. A working diagnosis of background H.pylori pangastritis with intestinal obstruction secondary to rectal polyp to rule malignancy or faecal impaction was made; and a differential diagnosis of peritonitis secondary to pelvic inflammatory disease was entertained.

She was subsequently admitted after abdominal X-ray, ultrasound, complete blood count, BUN, electrolytes and urea were requested. She was kept on nil per oral and placed on intravenous fluids; and parenteral rocephin and metronidazole.

The plain abdominal $\mathrm{x}$-ray showed air under the right hemidiaphragm and the abdominopelvic ultrasound scan revealed free mild fluid in the peritoneal cavity and lots of intestinal gas suspicious of pelvic inflammatory disease. The complete blood count, urea and electrolytes, BUN and creatinine were within normal range.

There was no significant improvement on the patient's condition as there was increasing distension of the abdomen and constipation. Therefore she was prepared for an emergency laparatomy .

At surgery, the following were found: a hard, annular and constricting rectosigmoid tumour; caecal rupture with attached bleeding tissue; faecal matter in the peritoneal cavity and gaseous distension of large bowel.

Subsequently, a near total colectomy was done, peritoneal cavity irrigated adequately and the ileal stump anastomosed to the anal stump and hemostasis secured.

Post operation condition of the patient improved satisfactorily and was discharged 9 days post surgery on antibiotics and haematinics. Periodic follow up at the surgical outpatient has been very satisfactory till date.

Gross examination of the colectomy specimen showed a segment of bowel measuring $12 \mathrm{~cm}$ in length and $7 \mathrm{~cm}$ in widest diameter. Cut section showed multiple greyish brown haemorrhagic masses on the mucosa surface measuring $2 \mathrm{~cm}$ $3 \mathrm{~cm}$ in diameter at the distal segment and an area of rupture at the proximal segment. Histology sections of the areas of rupture and tumour showed intestinal tissue composed of numerous islands of endometrial tissue with endometrial glands lined by columnar epithelium and surrounded by cellular endometrial stroma involving the entire wall. There is associated peri-lesional chronic inflammatory response. A histological diagnosis of multifocal intestinal endometriosis was made (Figures 1, 2, 3).

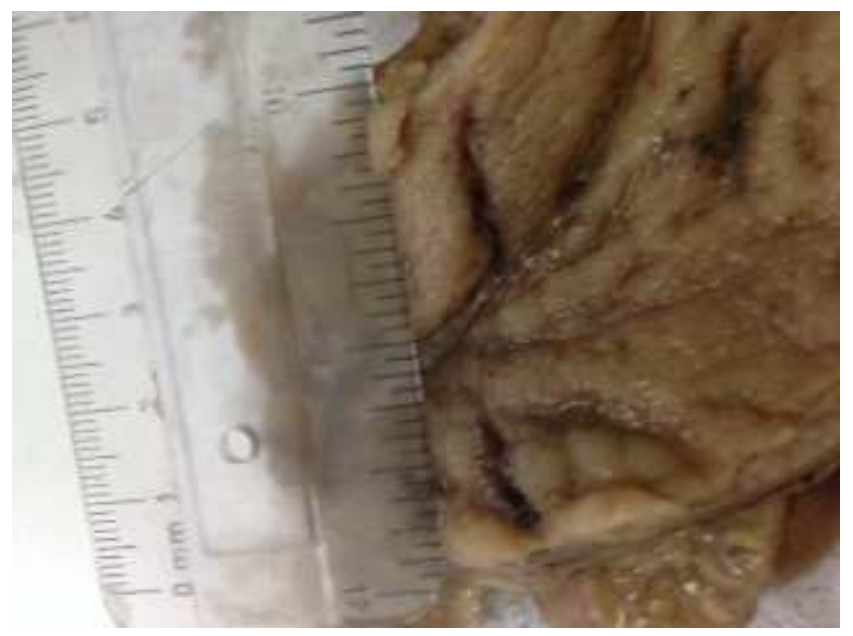

Figure 1. Bowel mucosa showing multifocal areas of endometriotic nodules.

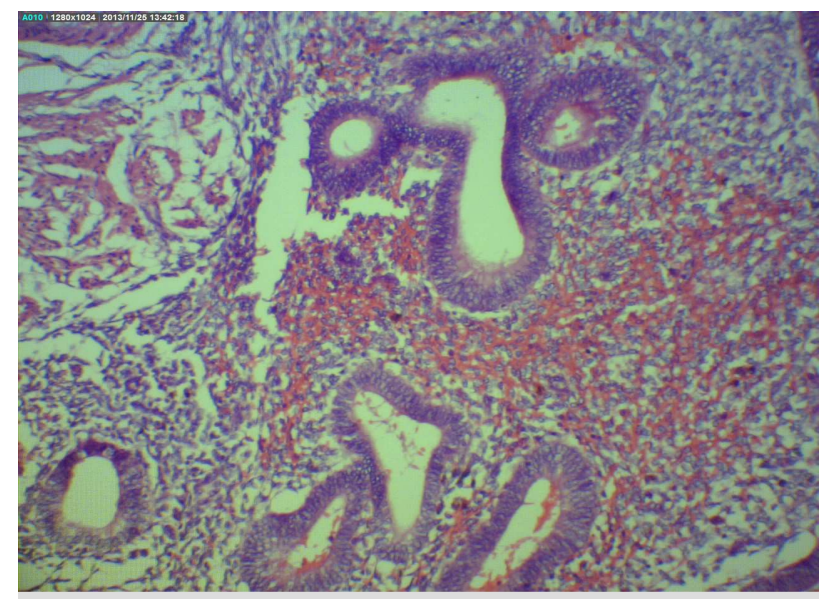

Figure 2. Section showing proliferative phase endometrial glands and stroma with areas of haemorrhage and chronic inflammatory cells (x100)

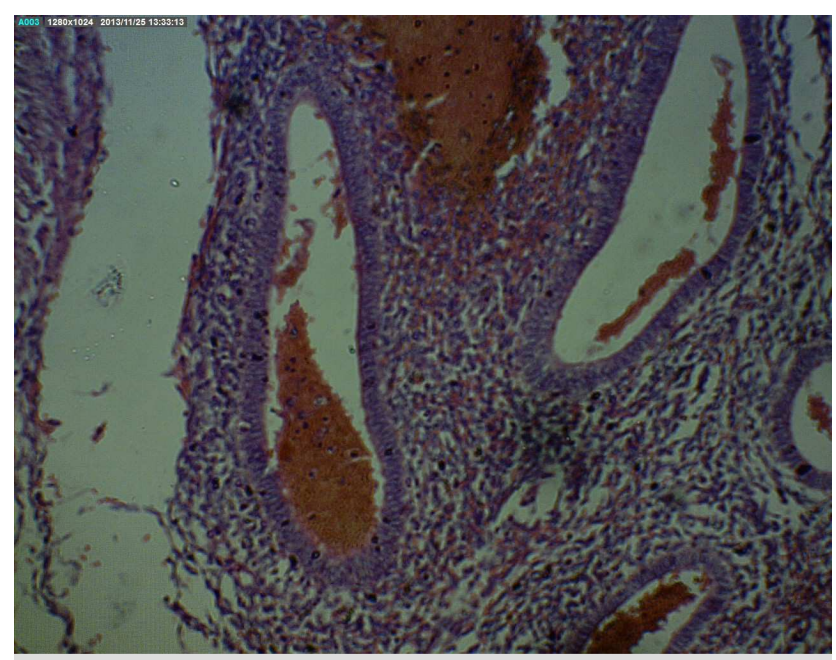

Figure 3. Section showing dilated endometrial glands containing haemorrhagic materials; and loosely cellular stroma. (x100)

\section{Discussion}

Intestinal endometriosis with ceacal involvement is rare while the rectosigmoid intestinal segment is mostly affected ${ }^{10}$. 
Multifocal involvement as seen in our patient has been observed in $15 \%-35 \%$ of cases. A review of 7000 patients with endometriosis shows an incidence of $4 \%$ and $70-88 \%$ for caecal and rectosigmoid segment respectively ${ }^{11}$. Likewise a 12 year study found appendix and ileocaecal involvement in $6.4 \%$ and $4.1 \%$ respectively of intestinal cases ${ }^{12}$. There is a strong association of bowel endometriosis with pelvic endometriosis; however, reports of bowel endometriosis independent from pelvic disease exist. This is the case in our report of an isolated bowel endometriosis ${ }^{12}$.

The aetiology of endometriosis remain unknown and controversial $^{13,14}$. Many theories have been proposed to explain this condition. Sampson's retrograde menstruation theory of endometrial tissue refluxes through the fallopian tubes, implanting and growing on the serosal surface of the abdominal and pelvic organs ${ }^{15,16}$ is the most widely accepted. Other theories include Minh's which states that endometrial tissue could occur as a result of metaplastic transformation of pluripotential peritoneal mesothelium ${ }^{17}$. Donne et $\mathrm{al}^{18}$ hypothesized that endometrial nodules may develop from metaplasia of mullerian implants. Another theory implies the migration of cells through the lymphatic or haematogenous spread $^{18}$. The new concept in endometriosis formation is the 'neurologic hypothesis' that the lesions seem to infiltrate the large bowel wall along the nerves at a distance from the primary lesion ${ }^{19}$. Other factors however, like genetic, hormonal and immune factors have also been postulated to increase susceptibility of some womaen to endometriosis ${ }^{20}$. Based on the finding of aromatase cytochrome p450 in endometriotic tissue but not in normal endometrium, it has been suggested that endometriotic tissue possesses the capacity to produce its own oestrogen via this enzyme ${ }^{21}$.

Other reports indicate that endometriosis is clonal in origin suggesting important biochemical differences between endometriotic tissue and normal uterine edometrium ${ }^{1}$.

Establishing a preoperative diagnosis of GI endometriosis is fraught with danger because of the possibility of a wide spectrum of diseases that its GI tract symptoms mimic. These include among others, infectious diseases, irritable bowel syndrome, inflammatory bowel disease and neoplasms ${ }^{22,23,24,25}$. It presents with bouts of abdominal pains, abdominal distension, tenesmus, constipation and diarrhoea ${ }^{26}$ Our patient presented with recurrent episodes of colicky abdominal pain, constipation and increasing abdominal distension and tenderness with vomiting. Compared with previous reports, it was difficult in our case to establish an accurate preoperative diagnosis because the symptoms were non-specific and could be due to other causes of bowel obstruction. Endometriosis should be suspected in a woman of reproductive age, nulliparous with abdominal pains in conjunction with signs of intestinal obstruction ${ }^{27}$. Our patient is young (34 years) and nulliparous with signs and symptoms suggestive of bowel obstruction.

Our patient had an emergency laparotomy for her disease because of increasing abdominal pain and distension and inability to arrive at a definitive diagnosis.

Most patients with intestinal obstruction due to endometriosis usually receive surgical intervention, while others without obstruction can take hormones like danatol and GnRH-a, but if they suffered from change in bowel habit, abdominal pain, haematochecia, or even intestinal obstruction, they usually receive surgical treatment ${ }^{26,28,29}$.

Conclusion

We have reported an uncommon presentation of intestinal endometriosis with large bowel obstruction in a nulliparous lady with infertility. Preoperative diagnosis of endometriosis will continue to pose a challenge as the signs are non-specific and it should be considered in patients in reproductive age with abdominal or pelvic pain in addition to signs of obstruction.

\section{References}

[1] Clement PB, Young RH, Scully RE. Stromal endometriosis of the cervix: a variant of endometriosis that may simulate a sarcoma. Am J Surg Pathol 1990; 14:449-455.

[2] Batt, Ronald E. A history of endometriosis. London: Springer. 2011;13-38

[3] Stratton P, Berkley KJ. "Chronic pelvic pain and endometriosis: translational evidence of the relationship and implications". Hum. Reprod. Update 2011;17(3): 327-46

[4] Samper ER, Slagle GW, Hand AM. Colonic endometriosis:its clinical spectrum. South Med J 1984; 77:912-4.

[5] Croom RD 3rd, Donovan ML, Sshwesinger WH. Intestinal endometriosis. Am J Surg 1984; 148:660-7.

[6] Lin YH, Kuo LI, Chuang AY, Cheng TI, Hung CF. Extrapelvic endometriosis complicated with colonic obstruction. J Chin Med Assoc 2006, 69:47-50.

[7] Macafee $\mathrm{CH}$, Greer HL. Intestinal endometriosis. J obstet Gynaec Brit Emp 1960; 67:539-55.

[8] Kratzer G, Salvati E. Collective review of endometriosis of the colon. Am J Surg 1955; 90:866-9.

[9] Zwas FR, Lyon DT. Endometriosis. An important condition in clinical gastroenterology. Dig Dis Sci 1991; 36:353-64.

[10] Alistair AP Slesser, Sufian Sultan, Faris Kubba, Davoid P Sellu. Acute small bowel obstruction secondary to intestinal endometriosis, an elusive condition: a case report. World Journal of Emergency Surgery 2010, 5:27.

[11] Szucs RA, Turner MA. Gastrointestinal tract involvement by gynaecologic diseases. Radiographics 1996, 16(6):1251-70.

[12] Chapron C, Chopin N, Borghese B, Foulot H, Dousset B, Vacher-Lavenu MC, Viera M, Hasan M, Bricou A. Deeply infiltrating endometriosis: pathogenetic implications of the anatomical distribution. Hum Reprod 2006, 27(7):1839-45.

[13] Redwine DB. Ovarian endometriosis: a marker for more extensive pelvic and intestinal disease. Fertil Steril. Aug 1999;72(2):310-315

[14] Scarmato VI, Levine MS, Herlinger H, Wickstrom M, Furth EE, Tureck RW. Ileal endometriosis: radiographic findings in 5 cases. Radiology 2000, 214(2):509-12. 
[15] Popoutchi P, dos Reis Lemos CR, Silva JC, Nogueira AA, Feres O, Ribeiro da Rocha JJ. Postmenopausal intestinal obstructive endometriosis: case report and review of the literature. Sao Paulo Med J 2008, 126(3):190-3.

[16] Quinn M. Endometriosis: the consequence of neurologic dysfunction. Med hypotheses 2004; 63:602-608.

[17] Witz CA. Current concepts in the pathogenesis of endometriosis. Clin Obstet Gynecol. 1999; 42:566-585.

[18] Mihn HN, Smadja A, Orcel L. (An integrated histogenetic concepts of internal and external endometriosis) L Gynecol Obstet Biol Reprod (Paris) 1986; 15:29-35.

[19] Donner J, Spada F, Squifflet J, Nissole M. Bladder endometriosis must be considered as bladder adenomyosis. Fertil Steril 2000; 74:1175-1181.

[20] Audebert AJ. (External endometriosis histogenesis, etiology and natural course). Rev Prat. 1990; 40:1077-1081.

[21] Anaf V, El Nakadi I, Simon P, van de Stadt J, Fayt I, Simonart T, Noel JC. Preferential infiltration of large bowel endometriosis along the nerves of the colon. Hum Reprod. 2004; 19:996-1002.

[22] Yantiss RK, Clement PB, Young RH. Endometriosis of the intestinal tract: a study of 44 cases of a disease that may cause diverse challenges in clinical and pathologic evaluation. Am J Surg Pathol. 2001; 25:445-454.
[23] Cappel MS, Friedman D, Mikhail N. Endometriosis of the terminal ileum simulating the clinical, roentgenographic, and surgical findings in Crohn's disease. Am J Gastroenterol. 1991; 86:1057-1062.

[24] Dimoulios P, Koutrobakis IE, Tzardi M, Antoniou P, Matalliotakis IM, Kouroumalis EA. A case of sigmoid endometriosis difficult to differentiate from colon cancer. BMC Gastroenterol. 2003; 3:18.

[25] Remorgida V, Ferrero S, Fulcheri E, Ragni N, Martin DC. Bowel endometriosis: presentation, diagnosis, and treatment. Obstet Gynecol Surv. 2007;62:461-470

[26] Orbach IK, Reich H, Orbach M, Orbach L. Laparascopic treatment of recurrent small bowel obstruction secondary to ileal endometriosis. J Minim Invasive Gynecol. 2007; 14:113115 .

[27] Antonella De Ceglie, Claudio Bilardi, Sabrina Blanchi, Massimo Picasso, Marcello Di Muzio, Alberto Trimarchi, Massimo Conio. Acute small bowel obstruction caused by endometriosis: A case report and review of literature. World J Gastroenterol. 2008; 14(21):3430-3434.

[28] Boadech JM. Endoscopic diagnosis of colonic endometriosis. Gastrointest Endosc. 1992; 38:568-580.

[29] Baong Peng, Jianhua Qian, Xiaoyun Wan, Chang Su. Endometriosis induced acute intestinal obstruction: A case report and literature review. Scientific Research and Essays. 2011;6(14):2976-2979. 